\title{
Application Value of High-Frequency Ultrasound and Contrast-Enhanced Ultrasound in Patients with Knee Osteoarthritis with Different TCM Syndromes
}

\author{
Yufei Wang1, Yingcong Xiao², Zhao Shen'2, Peilu Wang', He Chang', Hongjuan Mao, Xiufen Yao3, \\ Youmin Guo ${ }^{3 *}$ \\ ${ }^{1}$ Department of Imaging, Affiliated Hospital of the First Affiliated Hospital of Xi'an Jiaotong University, Xi'an, China \\ ${ }^{2}$ School of Medical Technology, Affiliated Hospital of Shaanxi University of Chinese Medicine, Xianyang, China \\ ${ }^{3}$ Department of Ultrasound, Affiliated Hospital of Shaanxi University of Chinese Medicine, Xianyang, China \\ Email: *cjr.guoyoumin@vip163.com
}

How to cite this paper: Wang, Y.F., Xiao, Y.C., Shen, Z., Wang, P.L., Chang, H., Mao, H.J., Yao, X.F. and Guo, Y.M. (2020) Application Value of High-Frequency Ultrasound and Contrast-Enhanced Ultrasound in Patients with Knee Osteoarthritis with Different TCM Syndromes. Journal of Biosciences and Medicines, 8, 149-156. https://doi.org/10.4236/jbm.2020.812014

Received: November 19, 2020

Accepted: December 19, 2020

Published: December 22, 2020

Copyright $\odot 2020$ by author(s) and Scientific Research Publishing Inc. This work is licensed under the Creative Commons Attribution International License (CC BY 4.0).

http://creativecommons.org/licenses/by/4.0/

\begin{abstract}
Objective: To explore the application value of high-frequency ultrasound and contrast-enhanced ultrasound in different syndrome types of knee osteoarthritis, and to provide more imaging evidence for clinical diagnosis and treatment. Method: Sixty patients with KOA were selected according to TCM classification, which were mainly divided into Qi stagnation and blood stasis type and cold-dampness blockage type. All knee joints were routinely examined by high frequency ultrasound, and those with synovial hyperplasia were examined by contrast-enhanced ultrasound. High frequency ultrasound is the examination of synovium, cartilage and collateral ligament of knee joint based on two-dimensional ultrasound. Contrast-enhanced ultrasound (CEUS) refers to the contrast examination of synovium in knee joint patients with synovial hyperplasia. Result: Among them, the suprapatellar sac effusion and synovial thickening of Qi stagnation and blood stasis type were more obvious than those of cold-dampness arthralgia type $(\mathrm{P}<0.05)$, and the degree of wear of the intercondylar cartilage of cold-dampness arthralgia type was more obvious than that of Qi stagnation. The blood stasis type is heavier and the meniscus bulge is higher $(\mathrm{P}<0.05)$. The radiography of synovial hyperplasia showed that the area under the curve of Qi stagnation and blood stasis type was higher than that of cold dampness arthralgia type $(\mathrm{P}<0.05)$, and the peak time was significantly shorter than that of cold dampness arthralgia type. There was no statistical difference in effective peak gradient and onset time of type $(\mathrm{P}>0.05)$. Conclusion: To a certain extent, the high-frequency ultrasound and contrast-enhanced ultrasound performance of knee osteoarthritis can be used
\end{abstract}


as a dialectical reference for different TCM syndrome types.

\section{Keywords}

Knee Osteoarthritis, High-Frequency Ultrasound, Contrast-Enhanced Ultrasound Syndrome

\section{Introduction}

Knee Osteoarthritis (KOA) is due to a variety of reasons that cause cartilage degenerative damage in the human knee joint, osteophytes on the edges of the joints, and narrow knee joint gaps, and it can also be accompanied by varying degrees of slippage. Knee osteoarthritis is mainly manifested as a chronic inflammatory disease of the synovium [1]. Knee osteoarthritis is mainly caused by the opening of the skin and hair, and the invasion of wind, cold and dampness, causing the meridians to become disharmony, stagnation of Qi and blood stasis, and become bone arthritis [2]. This disease is mainly characterized by deficiency of vital energy and weakness. It has a long course of disease and is difficult to cure because of repeated attacks [3]. This study will explore and analyze the relationship between the characteristics of the knee joint ultrasound image and the synovial performance of the knee joint under contrast-enhanced ultrasound and the TCM syndrome types of the knee joint, so as to provide a microscopic and visual diagnosis basis for KOA's TCM syndrome differentiation. The research results are reported as follows.

\section{Materials and Methods}

\subsection{Research Object}

Select the patients who came to the University Affiliated Hospital of Shaanxi Hospital of Traditional Chinese Medicine from June 2019 to June 2020 for examination and met the relevant diagnostic criteria of KOA and passed the TCM syndrome classification. Since two middle-aged doctors conduct independent dialectics, if the opinions are unified, use a unified opinion, if the opinions are not unified, the third senior doctor will dialectically.

\subsection{Diagnostic Criteria}

Diagnostic criteria of western medicine: diagnostic criteria of knee osteoarthritis formulated by American Rheumatology Society [4], diagnostic classification criteria of traditional Chinese medicine: refer to "Expert Diagnostic Consensus of Knee Osteoarthritis" "Therapeutics of Traditional Chinese Medicine" (2015 edition) [5], TCM syndrome differentiation belongs to Qi stagnation and blood stasis type and cold-dampness arthralgia type.

\subsection{Inclusion Criteria}

1) There is no history of trauma and pain in the knee joint recently; 
2) No rheumatism, fracture, infection and other diseases;

3) Age 30 - 60 years old, and volunteer to participate in this study.

\subsection{Exclusion Criteria}

1) Patients who have received other relevant treatments that may affect the observation of the indicators in this study;

2) Women during pregnancy or lactation;

3) Patients with severe life-threatening primary diseases such as cardiovascular, cerebrovascular, liver, kidney and hematopoietic system;

4) Mentally ill patients;

5) Patients with knee osteoarthritis caused by infection, rheumatoid arthritis and other diseases;

6) Patients who are unwilling to cooperate with this study.

\subsection{High Frequency Ultrasound Examination}

Use GELOGIQ E9 ultrasonic diagnostic instrument, select $9 \mathrm{MHz}$ probe, in musculoskeletal mode, take the patient supine position, knee flexion $90^{\circ}$, fully explode the knee joint, measure the knee suprapatellar sac effusion, the thickness of the hyperplastic synovium, the thickness of the intercondylar cartilage and the height of the meniscus.

\subsection{Contrast-Enhanced Ultrasound Examination Method}

For patients with synovial hyperplasia, synovial contrast was performed, and the ultrasound contrast agent was Shengnuowei, which was washed with $5 \mathrm{ml}$ of normal saline. On the basis of conventional ultrasound, choose the most obvious area of the anterior femoral synovial thickening as the observation site and record it, switch to the contrast-enhanced ultrasound mode, inject the agent, and turn on the timer at the same time to continuously observe the enhancement of the thickening slip in real time and save the image to be analyzed. Contrast analysis of contrast-enhanced ultrasound data, select a suitable position in the middle of synovial hyperplasia, obtain a time-intensity curve, observe all curve parameters, including peak time, area under the curve, effective peak gradient and starting time, and then analyze them.

\subsection{Statistical Analysis}

SPSS23.0 software was used to analyze the data, and t-test was used to compare the count data. $\mathrm{P}<0.05$ indicated that the difference was statistically significant.

\section{Results}

\subsection{Ultrasound Manifestations of KOA of Different Syndrome Types}

According to the classification of TCM syndromes of 60 knee joints, there were 26 cases of Qi stagnation and blood stasis type, and 39 cases of cold dampness 
arthralgia type. The suprapatellar bursa effusion, synovial membrane thickness, intercondylar cartilage thickness and meniscus height were compared. Table 1 shows that most affected knees are accompanied by different degrees of joint cavity effusion, mainly suprapatellar sac. Patients with Qi stagnation and blood stasis type have more suprapatellar sac effusions than those of cold-dampness-blocking type, and the depth is deeper $(\mathrm{P}<0.05)$, synovial hyperplasia is more obvious in $\mathrm{Qi}$ stagnation and blood stasis type, manifested by thickened synovium, unclear borders, increased echo, and increased blood flow signals. Comparing the thickness of the intercondylar cartilage, the abrasion is more prominent in the cold and damp arthralgia type, the cartilage surface is not smooth and rough, and the thickness is uneven. The protrusion height of meniscus bulging in the cold-dampness-blocking type was higher than that in the Qi stagnation and blood stasis type $(\mathrm{P}<0.05)$.

\subsection{Comparison of KOA Synovial Contrast Ultrasound with Different Syndrome Types}

High-frequency ultrasound showed that 24 cases of Qi stagnation and blood stasis type had synovial hyperplasia, and 36 cases of cold-dampness block type had synovial hyperplasia. The Qi stagnation and blood stasis type requires a shorter peak time and a larger area under the curve in CEUS. The gradient and time of arrival were not statistically different in knee osteoarthritis of cold-dampness blockage type and Qi stagnation and blood stasis type (Table 2).

Table 1. Ultrasonographic comparison of different types of knee osteoarthritis (mm).

\begin{tabular}{cccccc}
\hline Syndromes & $\mathrm{n}$ & $\begin{array}{c}\text { Suprapatellar } \\
\text { sac fluid }\end{array}$ & $\begin{array}{c}\text { Synovial } \\
\text { thickness }\end{array}$ & $\begin{array}{c}\text { Intercondylar } \\
\text { cartilage }\end{array}$ & $\begin{array}{c}\text { Meniscus } \\
\text { bulging }\end{array}$ \\
\hline Cold dampness & 39 & $1.73 \pm 1.51$ & $2.73 \pm 1.68$ & $1.53 \pm 0.29$ & $4.46 \pm 0.99$ \\
$\begin{array}{c}\text { Qi stagnation } \\
\text { and blood stasis }\end{array}$ & 26 & $5.17 \pm 2.10$ & $4.59 \pm 2.48$ & $2.49 \pm 0.42$ & $1.77 \pm 0.93$ \\
t value & -4.008 & -2.658 & -8.117 & -8.197 \\
P value & $<0.01$ & 0.010 & $<0.01$ & $<0.01$ \\
\hline
\end{tabular}

Table 2. Comparison of contrast-enhanced KOA synovial membrane with different syndrome types.

\begin{tabular}{cccccc}
\hline Syndromes & $\mathrm{n}$ & Peak time $(\mathrm{s})$ & $\begin{array}{c}\text { Area under the } \\
\text { curve }\left(\mathrm{cm}^{2}\right)\end{array}$ & $\begin{array}{c}\text { gradient } \\
(\mathrm{d}(\mathrm{dB}) / \mathrm{d}(\mathrm{t}))\end{array}$ & $\begin{array}{c}\text { Time of } \\
\text { arrival }(\mathrm{s})\end{array}$ \\
\hline $\begin{array}{c}\text { Cold dampness } \\
\begin{array}{c}\text { Qi stagnation and } \\
\text { blood stasis }\end{array}\end{array}$ & 24 & $43.43 \pm 12.57$ & $140.26 \pm 39.66$ & $0.53 \pm 0.75$ & $28.33 \pm 9.32$ \\
t value & $11.92 \pm 6.39$ & $416.43 \pm 292.07$ & $0.43 \pm 0.48$ & $29.85 \pm 5.51$ \\
P value & 8.537 & -3.983 & 0.408 & -0.541 \\
\hline
\end{tabular}




\section{Discussion}

\subsection{The Relationship between Knee Osteoarthritis and TCM Syndrome Types}

Regardless of physiologically or pathologically, the essence of knee osteoarthritis is the deficiency of liver and kidney [6]. Knee osteoarthritis is defined as a disease involving the entire joint, because it affects not only the cartilage, but also the changes in the subchondral bone and synovium [7]. Chinese medicine believes that the kidneys mainly control bones, and the liver and spleen mainly control muscles. Exercise can damage the liver and kidneys. Therefore, sports injuries and rheumatism can cause different types of knee pain. Cartilage degeneration and secondary bone hyperplasia are the main pathological features of knee osteoarthritis caused by cold-dampness arthritis [8]. Wind-cold-damp evil invades human body, causing poor circulation of Qi and blood, and stasis of muscles and veins. Therefore, the articular effusion and synovium of the cold-dampness block type show mild inflammation. Because of knee joint pain, the muscles near the joint produce protective spasms, which further restrict joint movement [9]. The hyperplasia of Qi stagnation and blood stasis synovitis occurs on the basis of articular cartilage degeneration, with joint capsule synovial damage, synovial congestion, and inflammatory exudation as the main pathological changes. Therefore, the articular cavity effusion and synovial hyperplasia of Qi stagnation and blood stasis knee osteoarthritis are more obvious.

\subsection{Distinguishing between Different TCM Syndromes of Knee Joints by Ultrasound}

This study also further verified the above point of view; the two types of knee osteoarthritis have statistical differences through high-frequency ultrasound and contrast-enhanced ultrasound analysis. For different syndrome types of knee osteoarthritis, there are statistical differences in suprapatellar sac effusion, thickness of synovial membrane, thickness of intercondylar cartilage and meniscus bulging. On the basis of bone hyperplasia, the cold-damp arthralgia type usually has cartilage degeneration and shedding. Ultrasound shows cartilage thinning and meniscus bulging are more prominent. Prolonged illness leads to liver and kidney deficiency, dystrophy of muscles and bones, and gradually twins and joint deformation [10]. Patients with Qi stagnation and blood stasis type have more joint cavity fluid, and synovial reactive hyperplasia is more obvious. The peak time and area under the curve are significantly different in the cold-dampness blockage type and the Qi stagnation and blood stasis type. The Qi stagnation and blood stasis type requires shorter peak time and larger area under the curve than the cold-dampness blockage type.

Synovial and cartilage lesions caused by the mutual influence of various factors in the joint cavity are one of the important links in the pathogenesis of knee osteoarthritis. In summary, the ultrasound examinations of the knee joints of the two types of KOA patients have different characteristic changes. Therefore, knee 
ultrasound examination and contrast-enhanced ultrasound examination can be used as an extension of the clinician's inspection, to explore the pathological changes of the internal tissue structure that the doctor can't see with the naked eye, and provide objective for the clinical differentiation and efficacy evaluation of KOA.

\subsection{Advantages Compared to MRI and X-Ray Ultrasound}

In the diagnosis of the knee joint, conventional X-ray is the most commonly used method for the diagnosis of knee joint disease, but it does not provide clinical anatomy such as articular cartilage, synovium, soft tissue in the joint cavity, and fluid-containing lesions. Especially when the lesions of knee osteoarthritis are mild, traditional imaging examinations are not sensitive to knee osteoarthritis [11]. With the continuous development and wide application of ultrasound medicine in clinical practice, especially in the field of musculoskeletal, high-frequency ultrasound provides other information on the pathological changes of soft tissues that cannot be described by conventional radiology (such as articular cartilage and synovium, meniscus) Injury, joint effusion, etc.). MRI is the gold standard for evaluating knee joints, and MRI is considered to be the most accurate imaging method for evaluating knee joints [12]. MRI has the advantages of non-invasiveness, multiplanarity, and good soft tissue contrast. However, MRI is expensive and cannot be performed for patients who have undergone cardiac bypass surgery. In recent years, due to the advantages of high-resolution ultrasound, such as dynamic and multi-plane, it has aroused great interest in the research and daily practice of knee osteoarthritis [13]. In the imaging technique for diagnosing knee osteoarthritis, musculoskeletal ultrasound has become a reliable one. Studies have shown that high-frequency ultrasound observation of knee arthritis synovial thickness, articular cartilage destruction and other pathological changes is comparable to X-ray examination. It has higher sensitivity, specificity and accuracy, and the examination of synovial blood flow is better than MRI examination [14]. High-frequency ultrasound and contrast-enhanced ultrasound can accurately detect joint effusions, show the lesions of synovium, cartilage, and subchondral bone, and are non-invasive, reproducible, intuitive, real-time, low-cost, rapid inspection, no radiation damage, and easy for patients The advantages of acceptance and follow-up inspection can also be carried out. It has obvious advantages in understanding the treatment effect of the disease and evaluating the curative effect. Therefore, high-frequency ultrasound is an important auxiliary examination method for the diagnosis of knee osteoarthritis.

In the imaging technique for diagnosing knee osteoarthritis, musculoskeletal ultrasound has become a reliable one. Studies have shown that high-frequency ultrasound observation of knee arthritis synovial thickness, articular cartilage destruction and other pathological changes is comparable to X-ray examination. Its sensitivity, specificity and accuracy are higher, and the examination of syn- 
ovial blood flow is also better than MRI examination. The sample size of this study is small, only for the two types of knee bones of cold-dampness blockage and Qi stagnation type. For arthritis, the curve value under contrast-enhanced ultrasound is only of reference value. In the future, the sample size needs to be expanded to explore the clinical significance of contrast-enhanced ultrasound for knee osteoarthritis of different TCM syndromes. In summary, high-frequency ultrasound and contrast-enhanced ultrasound can provide effective reference value for knee osteoarthritis of different TCM syndromes. High-frequency ultrasound and contrast-enhanced ultrasound are safe, low cost, reproducible, and have no obvious contraindications. Complications and other advantages are worthy of further study.

\section{Conflicts of Interest}

The authors declare no conflicts of interest regarding the publication of this paper.

\section{References}

[1] Lane, N.E., Shidara, K. and Wise, B.L. (2017) Osteoarthritis Year in Review 2016: Clinical. Osteoarthritis \& Cartilage, 25, 209-215. https://doi.org/10.1016/j.joca.2016.09.025

[2] Wang, F.N. (2020) Clinical Observation on Treating Knee Osteoarthritis of Blood Stasis Type with Acupotomy Combined with Warming Kidney and Activating Blood Circulation Recipe. Diet Health Care, 7, 103.

[3] Tu, Z.Y., Xiang, Y. and Luo, Y.X. (2019) Clinical Study on the treatment of Knee Osteoarthritis with Traditional Chinese Medicine Combined with Acupuncture and Massage. Chinese Medicine Modern Distance Education of China, 17, 57-58.

[4] Shi, G.Y. (2000) Overview of Arthritis. China Medical Science and Technology Press, Beijing, 339.

[5] Orthopedic Professional Committee of China Association for the Promotion of Chinese Medicine Research; Joint Working Committee of Orthopedics Professional Committee of Chinese Integrative Medicine Association (2015) Expert Consensus on Diagnosis and Treatment of Knee Osteoarthritis in Chinese Medicine (2015 Edition). Chinese Medicine Osteopathy, No. 7, 4-5.

[6] Yao, G.H., Liu, X.Q., Lu, M., et al. (2005) Research on the Diagnosis and Treatment Plan of Traditional Chinese Medicine Syndromes of Knee Osteoarthritis. Chinese Journal of Traditional Chinese Medicine, 23, 2125-2127.

[7] Loeser, R.F., Goldring, S.R., Scanzello, C.R., et al. (2012) Osteoarthritis: A Disease of the Joint as an Organ. Osteoarthritis, 64, 1697-1707.

https://doi.org/10.1002/art.34453

[8] Anonymous (2009) "Modern Knee Surgery" Has Been Published. Chinese Journal of Orthopaedics, 29, 1075-1075.

[9] Zheng, S.M. and Guan, J.H. (2012) A Clinical Study on the Relationship between TCM Syndromes of Knee Osteoarthritis and Its Arthroscopic Manifestations. Journal of Guangzhou University of Traditional Chinese Medicine, No. 4, 377-380.

[10] Zhang, D., Wang, Q.F., Yang, L.L., et al. (2018) Ultrasonographic Features and Correlation Study of Knee Osteoarthritis. China Orthopedics and Traumatology. 
[11] Cai, Y.Y., Feng, J.Z., Fang, Y.X., et al. (2016) Comparison of the Clinical Application Value of High-Frequency Ultrasound and X-Ray in Knee Osteoarthritis. Radiology Practice, 31, 42-445.

[12] Chen, Y.P., Chen, B., Zheng, Y.J., et al. (2017) Research Progress in the Diagnosis of Knee Osteoarthritis. Hunan Journal of Traditional Chinese Medicine, No. 5, 21.

[13] Hafezi-Nejad, N., Demehri, S., Guermazi, A., et al. (2018) Osteoarthritis Year in Review 2017: Updates on Imaging Advancements. Osteoarthritis \& Cartilage, 26, 341-349.. https://doi.org/10.1016/j.joca.2018.01.007

[14] Hayashi, D., Roemer, F.W. and Guermazi, A. (2016) Imaging for Osteoarthritis. Annals of Physical \& Rehabilitation Medicine, 59, 161-169.

https://doi.org/10.1016/j.rehab.2015.12.003 